\title{
Interactive proof-of-concept dashboard to explore patient follow-up in general practice
}

\author{
Robin De Croon, Joris Klerkx, Erik Duval \\ KU Leuven \\ Celestijnenlaan 200A \\ BE-3001 Heverlee \\ Email: robin.decroon@cs.kuleuven.be, joris.klerkx@cs.kuleuven.be, erik.duval@cs.kuleuven.be
}

\begin{abstract}
This paper reports on our proof-of-concept interactive visualization dashboard to support general practitioners (GPs) explore patient follow-up in their practice. The dashboard enables GPs to create visual queries in order to filter patients. Using the dashboard, GPs themselves can verify their practice with official quality indicators.
\end{abstract}

Keywords-general practice; dashboard; augment human intelligence

\section{INTRODUCTION AND RELATED WORK}

A follow-up is the act of seeing a patient to check the health status to determine changes or actions that took place after the patient's last visit. In health domains such as dentistry and physiotherapy, where regular meetings are common, it is clear which patients require a follow-up consultation. Patients should see their caregiver every $\mathrm{x}$ days, weeks, months or years. Yet, in general practice, some patients with chronic conditions are scheduled at fixed intervals, while other patients are often asked to take responsibility and call the doctor's practice themselves for a follow-up meeting. Sometimes, general practitioners (GPs) ask patients to phone in or attend a further check-up. This is not a reliable system as some patients fail to contact the practice [1], mainly because they do not consider a follow-up necessary. Recall systems to prevent these situations are often inadequate, preventing patients from getting their follow up tests and investigations. This results in missed or delayed diagnoses [1].

Patient follow-up quality is mainly studied in the context of hospitals. Most related literature discusses follow-up studies concerning a specific patient condition and somewhat neglects how to improve patient follow-up quality, specifically in general practice. Nevertheless, during an analysis of a GP's electronic medical record database with 4,184 patients, we discovered that some patients should, in fact, receive a new check-up.

GPs who want to gain insight into their follow-up capabilities sometimes request an audit from the Academic Center of General Practice. Unfortunately, not many GPs request such an audit as these are often perceived as a system to control the GP's work. We propose to empower GPs with an interactive dashboard where they themselves can query their data based on visual filters. The benefit of a visual solution is that GPs do not exclusively focus on a certain subset of patients and, thus, stay in control. Furthermore, it is impossible in a larger practice to contact all patients for all possible conditions. The present study is in line with previous research as described by Engelbart [2]: 'By 'augmenting human intellect' we mean increasing the capability of a man to approach a complex problem situation, to gain comprehension to suit his particular needs, and to derive solutions to problems." We aim to augment the intellect of the GPs by providing them with a tool enabling them to choose themselves to which group of patients to study.

Our dashboard is not the first medical visualization tool. Rind et al. (2013) [3] discuss fourteen state-of-the-art visual analytic tools designed to explore medical data stored in an electronic health record database. Yet, none of these tools focus specifically on the follow-up quality of patients. EventFlow is a well-known interactive visualization tool in this domain which displays and summarizes time-point and interval data. EventFlow groups individuals that sharing the same sequence of data points and displays the average interval times between events. The main goal of EventFlow is to reveal both common and rare patterns across the entire database [4]. Eventflow and the other existing tools focus on discovering general patterns in the data. Our dashboard differs from the existing solutions, since it is designed as an easy-to-use visual query tool to discover which patients need a follow-up and, only secondarily, tries to show relevant patterns.

\section{DESIGN}

Medidoc and HealthOne are the only two out of the fifteen homologated electronic medical record (EMR) systems in Belgium that offer statistical tools which can be used for follow-up insights. These tools only offer basic options to filter patients; for example, when a GP wants to filter patients based on medical parameters in Medidoc, it is only possible to do so based on length, weight, blood pressure and BMI. These are hard-coded queries for which no visual feedback is provided, as shown in Fig. 1. The GP first has to perform a query before the results are visible. These queries typically run for several seconds to minutes, which makes it harder for GPs to quickly explore their patient files and GPs thus need to know what they are looking for, whereas in our proof-of-concept, GPs immediately see if and how many patients are in the requested range. As explained in more detail in Section III, the visual queries are performed in near real-time, which makes it easier for GPs to interactively analyze their data. To improve the current workflow, our design follows Shneiderman's information seeking mantra [5]: overview first, zoom and filter, then details on demand. Initially, all patient data is visualized and GPs can manually 


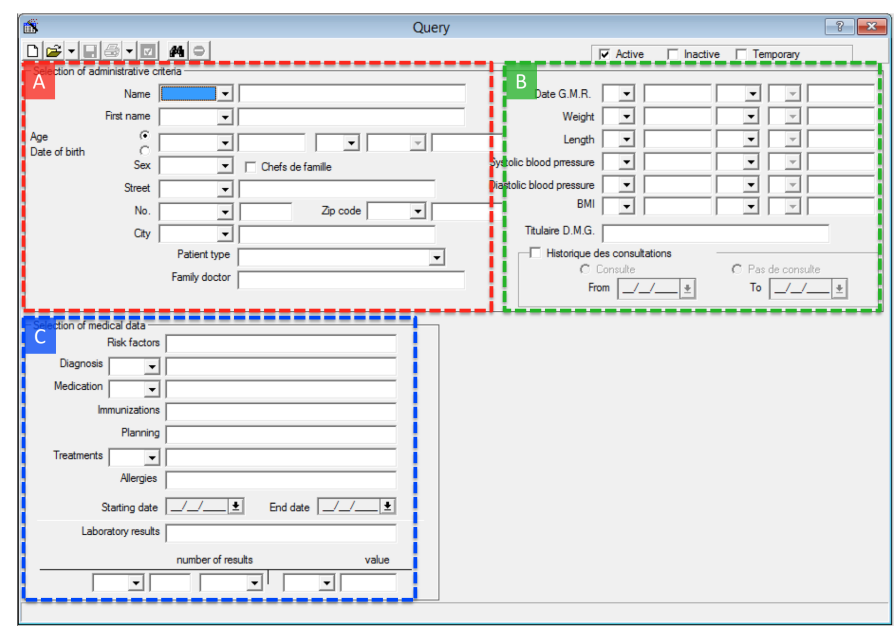

Fig. 1. Current statistical functions in one of the most used EMR software systems in Belgium. Part A enables the GP to filter patients based on demographic information. The GP can apply the filters based on medical parameters in Part B. Finally, in Part C, the GP can filter based on medical conditions.

filter patients based on their criteria. The design is represented by a dashboard consisting of several widgets that serve as visual filters or dynamic queries [6], as shown in Fig. 2

To actually improve follow-up quality, GPs should be able to quickly detect patients who require follow-up, such as patients with hypertension 1 , i.e. with blood pressure values above 140/90 $\mathrm{mmHg}$. Moreover, more conditions require proper follow-up, and, therefore no predetermined filters are set. GPs stay in control as the interactive visualizations only assist GPs to detect interesting ranges and patterns in their data.

Patient data is a collection of different data types and following Stevens's model [7], they can be categorized into tabular and relational data. Medical conditions are often related and this clearly reflects in the data. The tabular data consists of ordinal data, such as information on the main caregiver or medication, and of quantitative measurements, such as blood pressure or weight. Parameters can be measured by GPs themselves or by a laboratory. Systolic and diastolic blood pressure, heart rate, weight, temperature and peak-flow represent more than $90 \%$ of the parameters entered by GPs ${ }^{2}$

As can be seen in part A of Fig. 2, a parallel coordinates widget is added in order to quickly filter patients based on multivariate parameters. Parallel coordinates are a way to visualize and analyze multivariate data [8]. Each dimension of the data corresponds to vertical, uniformly spaced axes, and a patient is visualized as a connected set of points, one on each axis. This parallel coordinates widget visualizes most quantitative parameters of all patients. Besides being a space-efficient method to represent a large multi-variate data-set, the widget is also interactive. GPs can select the desired ranges and thus make a first selection. For example, the GP can select all patients with hypertension that did not show up for the previous 60 days. Thanks to the setup of parallel

\footnotetext{
${ }^{1}$ nlm.nih.gov/medlineplus/ency/article/000468.htm

${ }^{2}$ Based on our own analysis of a medical database with data from 4184 patients.
}

coordinates, it is clear how selected patients perform on other parameters as well. It is possible to filter patients based on multiple parameters simultaneously. The GP can decide which medical parameters determine the current selection. To facilitate the comparison among medical parameters, it is possible to reposition the horizontal layout of the axes. Every time the GP applies a visual filter by selecting a certain range on an axis, the other widgets immediately update to represent the selection.

Several studies show the correlation between population groups and the risk of certain conditions. For example, the National Center for Health Statistics in the USA has shown that the population groups with the highest drug poisoning death rates in 2008 were males, people aged 45-54 years, and non-Hispanic white and American Indian or Alaska Native persons ${ }^{3}$ This is why part B in Fig. 2 shows a demographic overview of the selection. These widgets allows the GP to easily filter on gender and age groups and the map widget adds geographical information to the dashboard. The heatmap visualizes the residency of the selected patients. Thus making it clear if there are problem areas in the GP's municipality. For example, geographical correlations can show if there is a flu outbreak in a certain region [9]. Furthermore, it enables the GP to select or contact every patient from that region or even contact patients from neighboring regions as a preventive precaution. Similarly, geolocation is used in an on-going study to show the prevalence of a chronic obstructive pulmonary disease in the proximity of a steel manufacturer.

Not all patient data are quantitative or demographic data. Medication is often a strong indicator for a follow-up visit. For example, female patients that take Roacutane should see their prescriber monthly ${ }^{4}$ This is why part D of the dashboard enables GPs to filter on ordinal data as well. This part shows common bar charts, which are formatted according to Tufte's design guidelines [10] in order to minimize visual literacy. It is also possible to filter patients based on conditions such as diabetes, which require a stricter follow-up policy 5 According to the Academic Center of General Practice, which regularly perform audits on general practices, secondary conditions and medication groups are important factors. For example, diabetic patients who also suffer from a heart condition should receive medication from another medication group than 'regular' patients.

Widget D in Fig. 2 lists the selected patients. GPs can choose to open the patient file, call the patient, send (custom) appointment reminders or flag a warning when the patient comes for another visit. The option to quickly contact the selected patients is added because a physical follow-up is not always needed. Even a quick phone call is associated with increased patient satisfaction and resolution of medication-related problems; a quick physical or virtual check-up may prevent health problems [11].

\section{IMPLEMENTATION}

The dashboard is a web-application implemented in JavaScript using state-of-the-art open source toolkits. The d3js

\footnotetext{
${ }^{3} \mathrm{http}: / / \mathrm{www} . c d c . g o v / \mathrm{nch} /$ data/databriefs/db81.htm

${ }^{4}$ http://www.drugs.com/pro/accutane.html

${ }^{5} \mathrm{nlm}$.nih.gov/medlineplus/ency/patientinstructions/000082.htm
} 


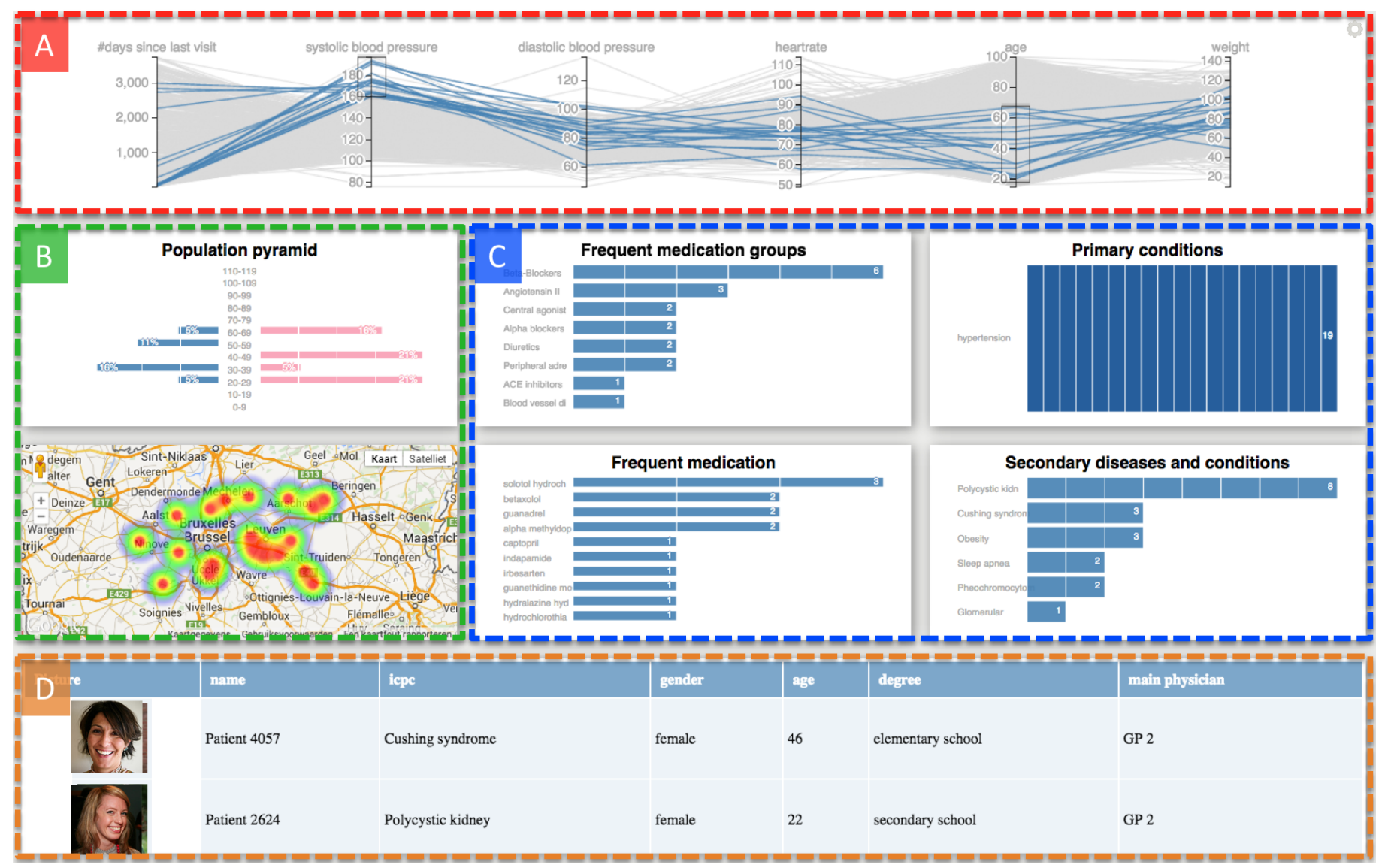

Fig. 2. Initially the data of all patients is visualized in the dashboard (overview first). Part A shows the parallel coordinates visualization where all patients with a systolic blood pressure higher than 160 and of legal working age $(18 \leq$ age $\leq 67)$ are selected. Each line represents a patient that matches both filters; the GP can adjust these filters by selecting an area on the representative axes. Furthermore, the GP can configure which parameters should be visualized in this widget. Part B shows the demographic information of the filtered patients. The population pyramid shows that $63 \%$ of the selected patients are female, whereas the heatmap visualizes that the selected patients live near Brussels. Part $\mathrm{C}$ shows the bar charts that serve as ordinal filters. Hypertension is selected as primary condition (dark blue bar). Part D lists the patients filtered by the visual queries (details on demand). Note: part A shows real medical data, whereas the other widgets contain random data for anonymity and demonstration purposes. All photos are copyrighted under a CC BY-NC-SA 2.0 license.

framework [12] is the basis for all visualizations. The parallel coordinate widget is built using the d3.parcoords toolkit ${ }^{6}$ The heatmap is built using the heatmap.js visualization library 7 on top of the Google Maps application programming interface (API) ${ }^{8}$ Thanks to the integration of the crossfilter library ${ }^{9}$ it is possible to filter the large multi-variate dataset in near real-time. Finally, as the dashboard is always shown in full window mode, gridster.j ${ }^{10}$ handles the layout of the dashboard on every screen size and resolution.

In order to hide the identity of the patients and to show profile-pictures under a creative commons license, the RandomUser ${ }^{11}$ API is used in the bottom widget.

\section{DISCUSSION}

The visualizations are designed using real semi-anonymized patient data from an EMR database

\footnotetext{
${ }^{6}$ https://syntagmatic.github.io/parallel-coordinates/

${ }^{7}$ http://www.patrick-wied.at/static/heatmapjs/

${ }^{8} \mathrm{https}: / /$ developers.google.com/maps/

${ }^{9} \mathrm{http} / / /$ square.github.io/crossfilter/

${ }^{10} \mathrm{http} / / /$ gridster.net/

${ }^{11} \mathrm{https} / / /$ randomuser.me/
}

that we obtained through a confidentiality agreement. Names and contact information are removed, but addresses are saved in order to detect patterns in the heatmap. The major difficulties we faced are identical to the five challenges as described by Bayley et al. [13]: 1) missing data; 2) erroneous data; 3) uninterpretable data; 4) inconsistencies and 5) data stored in noncoded text notes.

One of the strengths of parallel coordinates, when they are used interactively, is the ability to reveal meaningful multivariate patterns and comparisons [14]. However, no patterns could be detected in the single database we had access to. Nonetheless, the dashboard immediately revealed erroneous and uninterpretable data, which can trigger GPs to correct and update their patient data.

The perceived usefulness and usability of the dashboard is checked within the Academic Center for General Practice in Leuven. The proof-of-concepts suffers from an entry barrier as GPs are not familiar with parallel coordinates. Yet, once the researcher quickly explained the principle of parallel coordinates, the proof-of-concept is well received. The near real-time filtering and immediate visual feedback is perceived as an important advantage over the existing statistical tools. 


\section{CONCLUSION AND FUTURE WORK}

The proof-of-concept dashboard shows potential. It augments the existing solutions GPs use to gather insights about their follow-up quality. The dashboard was received enthusiastically in the Academic Center of General Practice in Leuven. Furthermore, it lowers the burden to actually perform an audit of the GP's practice. Using our dashboard, GPs themselves can verify their practice with official quality indicators (e.g. as listed by the National Health Service (NHS) [15]), which may lead to a better follow-up quality and higher patient satisfaction.

In order to detect possible patterns, the proof-of-concept dashboard will be adapted to work with the GP's own EMR database. The final version will be evaluated in practice and compared with 1) the statistical tools in Medidoc and 2) EventFlow. We aim to integrate the final design of the dashboard into real electronic medical record software in order to evaluate the usefulness in the wild. Furthermore, we will ask at least ten GPs to use both our dashboard and the existing solutions with the same data loaded, in order to perform a comparative study.

\section{ACKNOWLEDGEMENTS}

This research was funded under research grand IWT 120896. The authors would like to thank the GP that shared his database, Corilus for access to their products and the Academic Center for General Practice in Leuven for their extensive feedback.

\section{REFERENCES}

[1] S. Bird, "A GP's duty to follow up test results." Australian family physician, vol. 32, no. 1-2, pp. 45-6, 2003.

[2] D. C. Engelbart, "Augmenting Human Intellect: A Conceptual Framework," Air Force Office of Scientific Research, AFOSR-3233, http://www.dougengelbart.org/ pubs/augment-3906.html, 1962.

[3] A. Rind, "Interactive Information Visualization to Explore and Query Electronic Health Records," Foundations and Trends $\mathrm{R}$ in Human-Computer Interaction, vol. 5, no. 3, pp. 207-298, 2013.

[4] M. Monroe, R. Lan, J. Morales del Olmo, B. Shneiderman, C. Plaisant, and J. Millstein, "The challenges of specifying intervals and absences in temporal queries: a graphical language approach," in Proceedings of the SIGCHI Conference on Human Factors in Computing Systems - CHI '13. New York, New York, USA: ACM Press, 2013, p. 2349.

[5] B. Shneiderman, "The eyes have it: a task by data type taxonomy for information visualizations," in Proceedings 1996 IEEE Symposium on Visual Languages. IEEE Comput. Soc. Press, 1996, pp. 336-343.

[6] — - "Dynamic queries for visual information seeking," Software, IEEE, vol. 11, no. 6, pp. 70 - 77, 1994.

[7] S. S. Stevens, "On the Theory of Scales of Measurement." Science (New York, N.Y.), vol. 103, no. 2684, pp. 677-80, Jun. 1946.

[8] A. Inselberg and B. Dimsdale, "Parallel coordinates," in Human-Machine Interactive Systems. Springer, 1991, pp. 199-233.
[9] S. van Noort, M. Muehlen, H. Rebelo de Andrade, C. Koppeschaar, J. Lima Lourenço, and M. G. M. Gomes, "Gripenet: an internet-based system to monitor influenza-like illness uniformly across europe," Euro surveillance: European communicable disease bulletin, vol. 12, no. 7, p. E5-6, July 2007.

[10] E. Tufte, The Visual Display of Quantitative Information, 2nd ed. Cheshire: Graphics Press, Nov. 2001, vol. 2.

[11] V. Dudas, T. Bookwalter, K. M. Kerr, and S. Z. Pantilat, "The impact of follow-up telephone calls to patients after hospitalization," The American Journal of Medicine, vol. 111, no. 9, pp. 26-30, Dec. 2001.

[12] M. Bostock, V. Ogievetsky, and J. Heer, " $\mathrm{D}^{3}$ : Data-Driven Documents." IEEE transactions on visualization and computer graphics, vol. 17, no. 12, pp. 2301-9, Dec. 2011.

[13] K. B. Bayley, T. Belnap, L. Savitz, A. L. Masica, N. Shah, and N. S. Fleming, "Challenges in using electronic health record data for CER: experience of 4 learning organizations and solutions applied." Medical care, vol. 51, no. 8 Suppl 3, pp. S80-6, Aug. 2013.

[14] S. Few, "Multivariate analysis using parallel coordinates," Perceptual Edge, pp. 1-9, 2006.

[15] Department of Health, "The NHS Outcomes Framework 2014/15," Department of Health, Tech. Rep. November 2013, 2013. [Online]. Available: https://www.gov.uk/government/uploads/system/uploads/ attachment_data/file/256456/NHS_outcomes.pdf 